\title{
In vitro and ex vivo Measurement of Prophylactic Dabigatran Concentrations with a New Ecarin-Based Thromboelastometry Test
}

\author{
Mareike Kristina Körber ${ }^{a}$ Elisabeth Langer ${ }^{b, c}$ Martin Köhr ${ }^{a} \quad K^{2}$ laus-Dieter Wernecke ${ }^{d}$ \\ Wolfgang Korte ${ }^{\mathrm{e}}$ Christian von Heymann ${ }^{\mathrm{a}, \mathrm{f}}$ \\ ${ }^{a}$ Department of Anesthesiology and Intensive Care Medicine, Charité -Universitätsmedizin Berlin, Campus Virchow-Klinikum, Berlin, \\ Germany; \\ ${ }^{b}$ Labor Berlin - Charite Vivantes GmbH Berlin, Berlin, Germany; \\ ${ }^{\mathrm{c}}$ Institute for Laboratory Medicine, Clinical Chemistry and Pathobiochemistry, Charité - Universitätsmedizin Berlin, \\ Campus Virchow-Klinikum, Berlin, Germany; \\ dSOSTANA GmbH, Berlin, Germany; \\ e Center for Laboratory Medicine; and Hemostasis and Hemophilia Center St. Gallen, Switzerland; \\ ${ }^{f}$ Department of Anesthesiology, Intensive Care Medicine, Emergency Medicine and Pain Therapy, Vivantes Netzwerk für Gesundheit GmbH, \\ Klinikum im Friedrichshain, Berlin, Germany
}

\section{Keywords}

Dabigatran · Hemorrhage · Coagulation measurement

\section{Summary}

Background: An increasing number of oral anticoagulants has been approved, including dabigatran etexilate (DE). DE is a direct thrombin inhibitor that requires no routine monitoring, but, if necessary (e.g. urgent surgery etc.), the diluted thrombin time measured with Hemoclot $^{\circledR}$ has shown reliable results. So far, no point-of-care (PoC) assay is available to measure DE effects. The EcaTEM assay uses ecarin to initiate the coagulation cascade at the step of thrombin generation and measures the clotting time (CT) by thromboelastometry. Methods: This study investigated the correlation of the EcaTEM with standard laboratory assays in dabigatran-treated patients. Ten patients undergoing total hip or knee arthroplasty were included in the study. DE for thromboprophylaxis was started $4 \mathrm{~h}$ after surgery. Blood samples were taken before surgery as well as 2, 6 and $12 \mathrm{~h}$ after ingestion on the 3rd postoperative day. Dabigatran concentration (Hemoclot), activated partial thromboplastin time, thrombin time and CT EcaTEM were measured. Results: Only CT EcaTEM and Hemoclot showed a correlation > 0.75 for all measurements. Conclusion: CT EcaTEM appears a valid PoC method parameter to detect thrombin inhibition and thus the presence of dabigatran be- side diluted thrombin time at different concentration levels. This may represent an opportunity to identify the presence of dabigatran, e.g., in emergency situations.

(c) 2017 S. Karger GmbH, Freiburg

\section{Introduction}

Anticoagulation is essential to prevent thromboembolic events such as deep vein thrombosis, pulmonary embolism or stroke. Stroke caused by atrial fibrillation leads to a poor outcome with possible long-time disability and death. Despite a potential negative outcome, many patients remain without anticoagulation therapy even though they qualify for anticoagulation by CHA2DS2VASc score [1]. Until a few years ago, only vitamin $\mathrm{K}$ antagonists were available for long-term outpatient anticoagulation, requiring repeated laboratory controls, given a relatively small therapeutic window. For some years, another group of oral anticoagulants, the direct oral anticoagulants, are now approved, e.g., for the prevention of stroke in patients with atrial fibrillation [2].

Dabigatran etexilate (DE), a direct thrombin inhibitor, is approved for thromboprophylaxis after hip and knee replacement surgery [3] and stroke prevention in patients with atrial fibrillation [4]. DE is a prodrug which is activated after bioconversion in the

\section{KARGER}

(๑) 2017 S. Karger GmbH, Freiburg 
liver. Advantages of dabigatran include that no routine laboratory monitoring is necessary [5], less drug interactions [6], and a safety profile superior to warfarin [7]. Albeit that routine laboratory monitoring of the anticoagulant effect is not necessary, it is desirable to be able to identify and/or quantify DE in cases of urgent surgery or life-threatening bleeding. The diluted thrombin time using the Hemoclot ${ }^{\circledR}$ assay has shown reliable results when compared to standard coagulation assays [8].

A standard laboratory parameter such as the activated partial thromboplastin time (aPTT) can hint to the presence of DE at peak levels and the undiluted thrombin time can give information about the presence of high concentrations of the drug; such unspecific assays, however, are not useful if reliable information on the estimate dabigatran concentration is required [9]. For life-threatening emergencies [9], a point-of-care (PoC) assay to measure the effect of dabigatran and to decide if reversal is indicated would be helpful. The ECATEM is an assay that uses ecarin to initiate the coagulation cascade at the step of thrombin generation and measures the clotting time (CT) by thromboelastometry $\left(\right.$ ROTEM $^{\circledR}$; TEM International, Munich, Germany). This study investigated the correlation of thromboelastometry, including the ECA-TEM assay, and standard laboratory assays with dabigatran plasma concentration in dabigatran-treated patients.

\section{Material and Methods}

\section{Study Population}

This study was approved by the ethics committee of Charité - Universitätsmedizin Berlin, and an informed consent was obtained by the participating patients. We included 10 healthy patients (female and male) aged 18-75 years undergoing total hip or knee arthroplasty in general anesthesia. Patients with known disorder of the coagulation system or taking other anticoagulants or platelet inhibitors were excluded.

Samples were taken immediately after induction of anesthesia (without DE) as well as 2, 6, and $12 \mathrm{~h}$ after intake of DE. DE dosing was started $6 \mathrm{~h}$ after surgery using $110 \mathrm{mg}$, followed by $110 \mathrm{mg}$ twice daily from the first postoperative day on. Patient blood samples with DE were collected in 3.13\% citrate tubes on the3rd postoperative day when a steady state of DE was achieved.

\section{Rotational Thromboelastometry}

Rotational thromboelastometry was performed on ROTEM Delta devices (courtesy of TEM International GmbH, Munich, Germany). We used the InTEM and ExTEM assays, which induce coagulation via the intrinsic and the extrinsic pathway, respectively. As a thrombin-specific test, EcaTEM was used as an experimental assay. EcaTEM is a new thromboelastometric assay based on the fact that Ecarin converts prothrombin to meizothrombin before clot formation takes place; this is inhibited in the presence of a specific thrombin inhibitor. CT in the TEM assays was our primary outcome variable for all assays.

\section{aPTT and Hemoclot}

The analyses of all plasma samples were performed on an automated coagulowing reagents were used: STA aPTT reagent (aPTT reference range 26-40 s); STA-Thrombin ${ }^{\circledR}$ reagent (thrombin time reference range $<13 \mathrm{~s}$; heparin method); Hemoclot ${ }^{\circledR}$ (thrombin inhibitor assay) with dabigatran calibrators (from Coachrom, Mariaenzersdorf, Austria). aPTT and Hemoclot were secondary outcome variables of this study.

\section{Medication for Patients} lation analyzer (STA-R Evolution Analyzer, Stago, Parsipanny, USA). The fol-

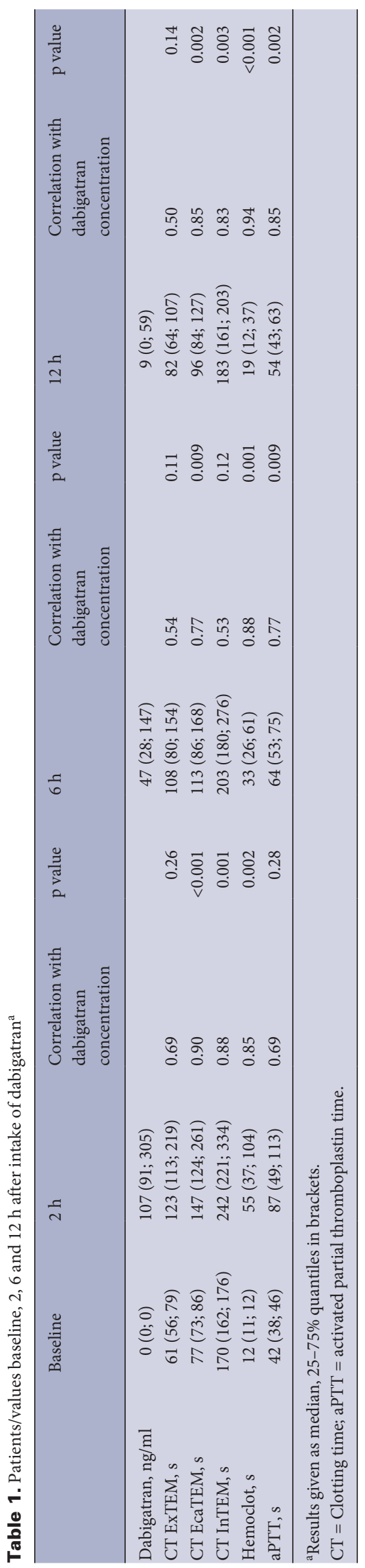

In vitro and ex vivo Measurement of Prophylactic

Transfus Med Hemother 2017;44:100-105 

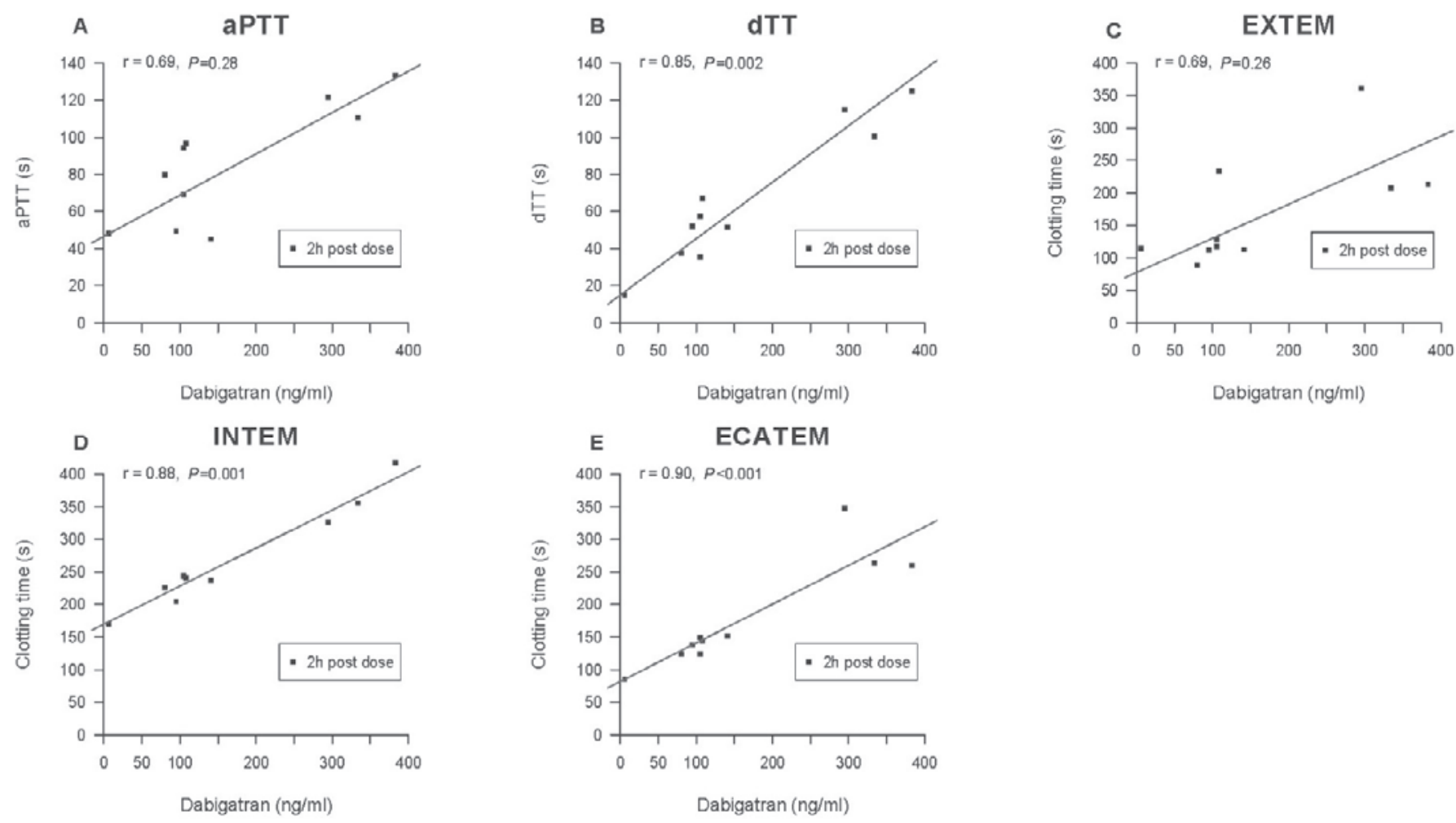

Fig. 1. Association of dabigatran plasma concentration $2 \mathrm{~h}$ post dose with standard coagulation variables (A aPTT and B diluted thrombin time) and CTs of advanced variables derived from thromboelastometry (C EXTEM, D INTEM, E ECATEM).

\section{Statistical Analysis}

Data are presented as median with $25-75 \%$ quantiles in brackets. The statistical evaluation contained an exploratory data analysis of the primary and secondary endpoints, including structural analyses. Assuming non-normal distribution of the observations, all statistical examinations were carried out as nonparametric tests (using the Mann-Whitney test for independent samples and Wilcoxon's pairwise test for paired observations). Observation of a $\mathrm{p}$ value of $<$ 0.05 (two-tailed) was considered to represent statistical significance. Statistical tests compared baseline values to each time point. Spearman's coefficient was used to evaluate the correlation between the investigated parameters.

The $\mathrm{p}$ values obtained are explorative, which is why no adjustment for multiple testing was made. Statistical evaluations was accomplished by using IBM ${ }^{\odot}$ SPSS $^{\complement}$ Statistics, Version 20 (SAS Institute, Inc., Cary, NC, USA).

\section{Results}

\section{Baseline Characteristics}

All patients showed values within the reference ranges for thromboelastometry and plasmatic coagulation tests, only the aPTT was slightly above the upper normal range with a median of 41.5 (37.6-47.1) s (table 1).

\section{DE Concentrations and Coagulation Studies}

We measured a median dabigatran concentration of 107 (91$305) \mathrm{ng} / \mathrm{ml}$ at $2 \mathrm{~h}, 47(28-147) \mathrm{ng} / \mathrm{ml}$ at $6 \mathrm{~h}$ and $9(0-59) \mathrm{ng} / \mathrm{ml}$ at $12 \mathrm{~h}$ after ingestion, which is within the expected range [6]. All parameters evaluated showed a significant prolongation (table 1). Only CT EcaTEM, CT InTEM and the Hemoclot correlated sig- nificantly with the measured dabigatran concentration, with a correlation coefficient above 0.75 . After $6 \mathrm{~h}$, CT EcaTEM, thrombin time, and aPTT showed a correlation coefficient $>0.75$, while after $12 \mathrm{~h}$ the CT EcaTEM, the aPTT, and the Hemoclot had the highest correlation coefficients (table 1). The distribution of measurements is shown in figures $1-3$.

\section{Discussion}

Our data show a highly significant correlation of a novel, thromboelastometry-based assay, the CT EcaTEM, over a wide range of plasma levels of dabigatran that seems to be comparable to the Hemoclot assay at all measured time points. The Hemoclot assay is still recognized as the gold standard for the measurement of dabigatran plasma levels. This diagnostic tool of the clinical laboratory may now be amended by the CT EcaTEM as a PoC test with results available in less than $10 \mathrm{~min}$. In the clinical setting, this could save precious time to start treatment in patients suffering bleeding complications with dabigatran medication, especially since idarucizumab is available. Idarucizumab, a monoclonal antibody that binds and thereby inactivates dabigatran, is available since 2016 for rescue reversal in case of severe bleeding or emergency surgery [10]. This is an important progress for dabigatran as an anticoagulant because it adds to its safety profile. A valid PoC method sensitive to dabigatran could save precious time in identifying dabigatran influence on the coagulation system and following 

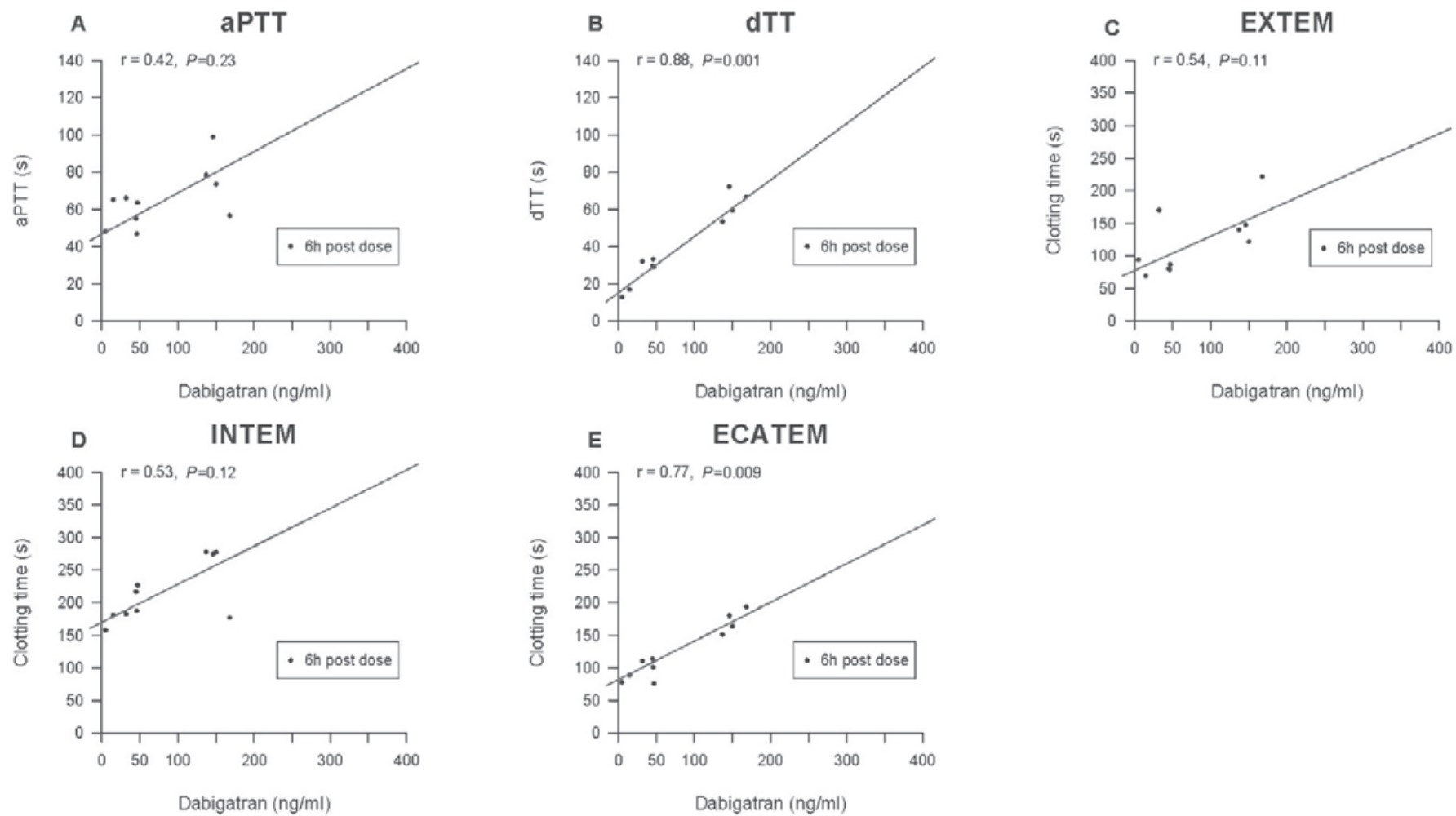

Fig. 2. Association of dabigatran plasma concentration $6 \mathrm{~h}$ post dose with standard coagulation variables (A aPTT and $\mathbf{B}$ diluted thrombin time) and CTs of advanced variables derived from thromboelastometry (C EXTEM, D INTEM, E ECATEM).
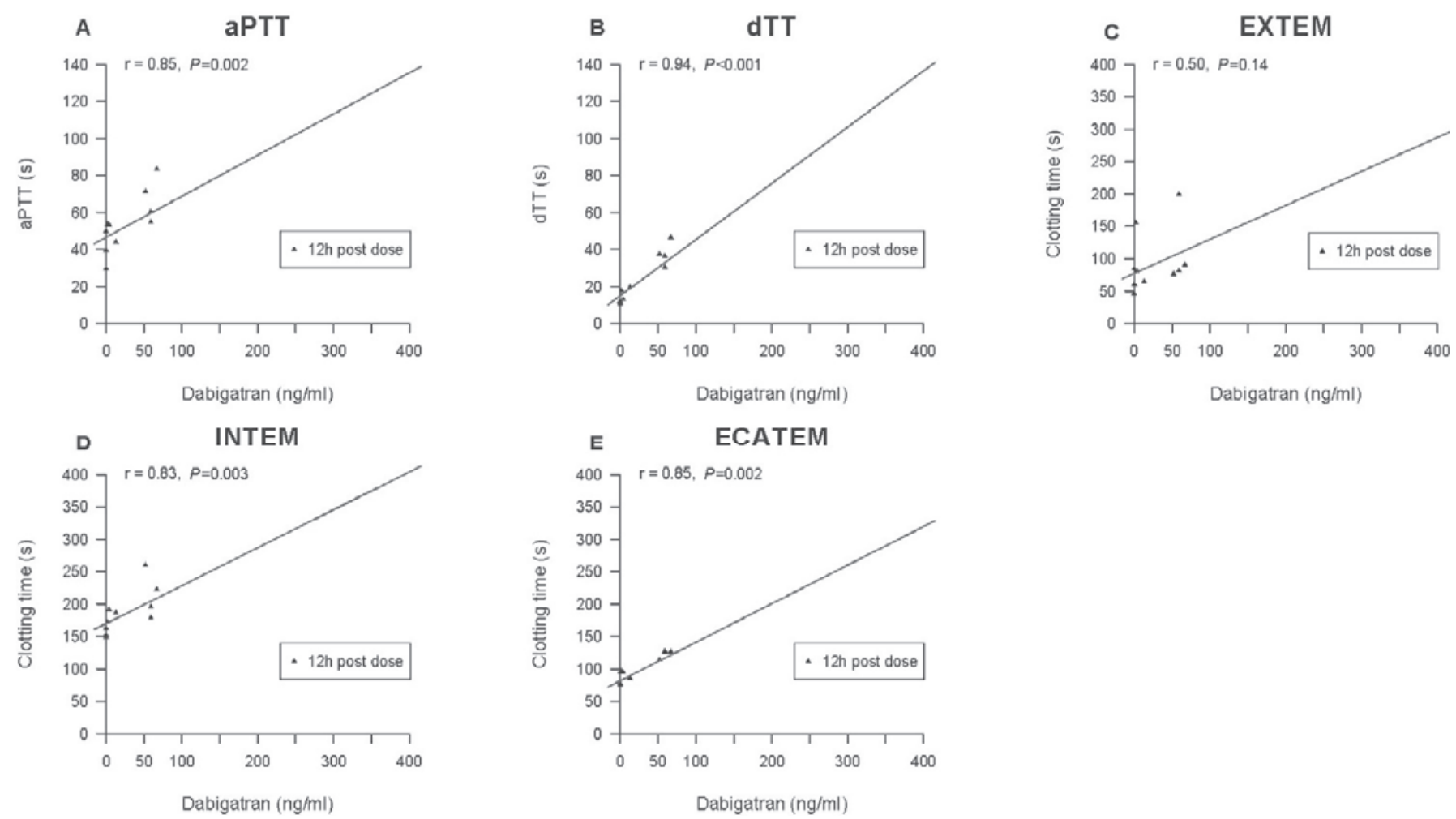

Fig. 3. Association of dabigatran plasma concentration $12 \mathrm{~h}$ post dose with standard coagulation variables (A aPTT and $\mathbf{B}$ diluted thrombin time) and CTs of advanced variables derived from thromboelastometry (C EXTEM, D INTEM, E ECATEM). 
up on its course once idarucizumab has been applied. The metabolism of dabigatran is stable in healthy patients. But as especially elderly patients may develop renal insufficiency or cognitive deficits with the risk of dabigatran overdosing - which might go unrecognized by outside caregivers [11] -, the importance of such a PoC method seems obvious.

\section{Measurement of Dabigatran with Thromboelastometry}

Two hours after dabigatran intake (i.e., at peak concentrations), the CT of all thromboelastometry parameters, except for CT ExTEM, were significantly prolonged and correlated well with prophylactic dabigatran concentrations measured with the calibrated Hemoclot test. This is consistent with the findings of Solbeck et al. [12] who investigated in vitro kaolin-activated thromboleastography with or without the addition of ecarin for measurement of dabigatran; they established a significant correlation for R-time (a parameter comparable to the $\mathrm{CT}$ parameter in thromboelastometry) with the Hemoclot test (0.891) and the ecarin CT (0.914) although they could not correlate the dabigatran concentration directly as they did not measure it. Dias et al. [13] evaluated the addition of ecarin to standard thromboelastography test assays and found a significant difference of R-time in the presence of dabigatran. These findings are supported by further investigations by Dias et al. [13] and by Davis et al. [14] who also found a prolongation of R-time through dabigatran. But as these experiments did not include dabigatran concentration measurements, they do not allow conclusions on the correlation of the assays evaluated with dabigatran concentrations [12-14]. Our results indicate that especially CT EcaTEM as a thrombin-sensitive parameter gives reliable results over a wide range of dabigatran concentrations comparable to the gold standard diluted thrombin time, ecarin CT, and dabigatran-calibrated thrombin time. This includes presumably therapeutic dabigatran concentrations as some patients show values nearly up to $300 \mathrm{ng} / \mathrm{ml}$ at $2 \mathrm{~h}$. The correlation of CT EcaTEM to DE concentration was between 0.77 and 0.9 at $2-12 \mathrm{~h}$ after dabigatran intake.

In emergency situations, the EcaTEM measurement can be performed at the bedside and thus does not necessarily requires transport of the sample to a specialized laboratory, as does the Hemoclot, which is not available in all hospitals. The resulting shorter turnaround times (5-10 min vs. 30-45 min for ecarin CT vs. diluted thrombin time) may allow for an expedited diagnosis in order to initiate emergency treatment. This would be in line with current guidelines for the management of bleeding events or ur- gent surgery, which recommend laboratory testing of the dabigatran concentration and dabigatran effects prior to reversal [15-19].

Measurement of Dabigatran with aPTT, Thrombin Time, and Dabigatran-Calibrated Thrombin Time

Our results show a good correlation between the assays evaluated and the dabigatran-calibrated diluted thrombin time for all measurements. There is variation for the correlation between aPTT and dabigatran-calibrated diluted thrombin time, depending on how long after the dabigatran intake the respective sample was taken $(2-12 \mathrm{~h}$, Pearson coefficient $0,69-0,85)$. This is in line with earlier reports suggesting less reliability of the aPTT for the detection of dabigatran [20]. In contrast, a dabigatran-calibrated diluted thrombin time is reliable but not readily available in every laboratory. The better correlation of the thrombin time over aPTT with dabigatran plasma levels was described by various investigators [21-24], which also might be explained by the fact that the aPTT results are highly dependent on the test reagent used [24,25].

\section{Study Limitations}

This is an ex vivo study in patients with total hip or knee arthroplasty. Thus, our results represent those of a pilot study that needs to be confirmed in a larger number of patients.

\section{Conclusions}

Our results suggest that a thrombelastometry-based wholeblood ecarin CT assay (CT EcaTEM) is able to detect dabigatran in a PoC setting and in a wide concentration range $(0-305 \mathrm{ng} / \mathrm{ml})$. With this PoC approach, it seems that detection of dabigatran and initiation of respective treatment measures, as appropriate, might be sped up considerably, possibly improving the patient's outlook for a beneficial outcome.

\section{Disclosure Statement}

Parts of the results were presented at ESA 2015 and ESICM 2015. CvH received honoraria for lectures and/or consultancy work related to this study from Pfizer GmbH, Bayer AG, Boehringer Ingelheim, Bristol Myers Squibb, NovoNordisk GmbH, Daiichi Sankyo GmbH, CSL Behring, TEM International $\mathrm{GmbH}$, and HICC GbR.

This study was funded by an unrestricted research grant by Boehringer Ingelheim Pharma GmbH \& Co. KG (Ingelheim am Rhein, Germany).

\section{References}

1 Mason PK, Lake DE, DiMarco JP, et al: Impact of the $\mathrm{CHA}_{2} \mathrm{DS}_{2}$-VASc score on anticoagulation recommendations for atrial fibrillation. Am J Med 2012;125:603. e1-e6

2 Ha AC, Verma A, Verma S: Oral anticoagulation for stroke prevention amongst atrial fibrillation patients with valvular heart disease: an update. Curr Opin Cardiol 2017;32:174-180.
3 Schulman S, Kearon C, Kakkar AK, Mismetti P, Schellong S, Eriksson H, Baanstra D, Schnee J, Goldhaber SZ; the RE-COVER Study Group: Dabigatran versus warfarin in the treatment of acute venous thromboembolism. N Engl J Med 2009;361:2342-2352.

4 Huber K, Connolly SJ, Kher A, et al: Practical use of dabigatran etexilate for stroke prevention in atrial fibrillation. Int J Clin Pract 2013;67:516-526.

5 Anklesaria Z, Al-Helou G: The new era of anticoagulation. Surg Neurol Int 2016;7:22.
6 Stangier J, Clemens A: Pharmacology, pharmacokinetics, and pharmacodynamics of dabigatran etexilate, an oral direct thrombin inhibitor. Clin Appl Thromb Hemost 2009; 15(suppl 1):9S-16S

7 Carmo J, Moscosco CF, Ferreira J, et al: Dabigatran in real-world atrial fibrillation. Meta-analysis of observational comparison studies with vitamin $\mathrm{K}$ antagonists. Thromb Haemost 2016;116:754-763. 
8 van Ryn J, Stangier J, Haertter S, et al: Dabigatran etexilate - a novel, reversible, oral direct thrombin inhibitor: interpretation of coagulation assays and reversal of anticoagulant activity. Thromb Haemost 2010;103: 1116-1127.

9 Thibault N, Morrill AM, Willett KC: Idarucizumab for reversal of dabigatran-induced anticoagulation: a systematic review. Am J Ther 2016;DOI: 10.1097/ MJT.0000000000000460.

10 Solbeck S, Meyer MA, Johansson PI, et al: Monitoring of dabigatran anticoagulation and its reversal in vitro by thrombelastography. Int J Cardiol 2014;176:794799.

11 Bansal N, Xie D, Tao K, et al: Atrial fibrillation and risk of ESKD in patients with CKD. Clin J Am Soc Nephrol 2016;11:1189-1196.

12 Solbeck S, Ostrowski SR, Stenballe J, et al: Thrombelastography detects dabigatran at therapeutic concentrations in vitro to the same extent as gold-standard tests. Int J Cardiol 2016;208:14-18.

13 Dias JD, Norem K, Doorneweerd DD, Thurer, RL Popovsky MA, Omert LA: Use of thromboelastography (TEG) for detection of new oral anticoagulants, Arch Pathol Lab Med 2015;139:665-673.
14 Davis PK, Musunuru H, Walsh M, Mitra R, Ploplis V, Castellino FJ: The ex vivo reversibility of dabigatraninduced whole-blood coagulopathy as monitored by thromboelastography: mechanistic implications for clinical medicine. Thromb Haemost 2012;108:586-588.

15 Dager W, Hellwig T: Current knowledge on assessing the effects of and managing bleeding and urgent procedures with direct oral anticoagulants. Am J Health Syst Pharm 2016;73(suppl 2):S14-26.

16 Gulseth MP: Overview of direct oral anticoagulant therapy reversal. Am J Health Syst Pharm 2016;73 (suppl 2):S5-13.

17 Sarma A, Guigliano RP: Current and developing strategies for monitoring and reversing direct oral anticoagulants in patients with non-valvular atrial fibrillation. Hosp Pract 2015;43:258-267.

18 Rossaint R, Bouillon B, Cerny V, et al: The European guideline on management of major bleeding and coagulopathy following trauma. Crit Care 2016;20:100.

19 von Heymann C, Rosenthal C, Kaufner L, Sander M: Management of direct oral anticoagulants-associated bleeding in the trauma patient. Curr Opin Aneaesthesiol 2016;29:220-228.
20 Wong WH, Yip CY, Sum CL, et al: A practical guide to ordering and interpreting coagulation tests for patients on direct oral anticoagulants in Singapore. Ann Acad Med Singapore 2016;45:98-105.

21 Cuker A: Laboratory measurement of the non-vitamin $\mathrm{K}$ antagonist oral anticoagulants: selecting the optimal assay based on drug, assay availability, and clinical indication. J Thromb Thrombolysis 2016;41:241-247.

22 Harenberg J: Laboratory determination of old and new targeted anticoagulant agents for prevention of bleeding and thrombotic events in cancer patients. Thromb Res 2016;140(suppl 1):S165-167.

23 Lippi G, Favaloro E: Recent guidelines and recommendations for laboratory assessment of the direct oral anticoagulants (DOACS): is there consensus? Clin Chem Lab Med 2014;53:185-197.

24 Van Blerk M, Bailleul E, Chatelain B, DeMulder A, Devrees K: Influence of dabigatran and rivaroxaban on routine coagulation assays: a nationwide Belgian survey. Thromb Haemost 2015;113:154-164.

25 Ramos-Esquivel A: Monitoring anticoagulant therapy with new oral agents. World J Methodol 2015;5:212215. 